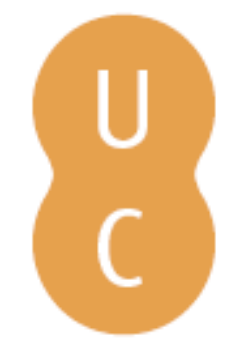

\title{
nommalina
}

\section{Novas ecologias do controlo bibliográfico: uma perspetiva sobre os futuros das bibliotecas e da informação bibliográfica nos meios digitais}

\author{
Autor(es): $\quad$ Lopes, António Tavares
}

Publicado por: Imprensa da Universidade de Coimbra

URL

persistente: URI:http://hdl.handle.net/10316.2/36987

DOI: $\quad$ DOI:http://dx.doi.org/10.14195/978-989-26-1045-0_16

Accessed : $\quad$ 26-Apr-2023 06:56:42

A navegação consulta e descarregamento dos títulos inseridos nas Bibliotecas Digitais UC Digitalis, UC Pombalina e UC Impactum, pressupõem a aceitação plena e sem reservas dos Termos e Condições de Uso destas Bibliotecas Digitais, disponíveis em https://digitalis.uc.pt/pt-pt/termos.

Conforme exposto nos referidos Termos e Condições de Uso, o descarregamento de títulos de acesso restrito requer uma licença válida de autorização devendo o utilizador aceder ao(s) documento(s) a partir de um endereço de IP da instituição detentora da supramencionada licença.

Ao utilizador é apenas permitido o descarregamento para uso pessoal, pelo que o emprego do(s) título(s) descarregado(s) para outro fim, designadamente comercial, carece de autorização do respetivo autor ou editor da obra.

Na medida em que todas as obras da UC Digitalis se encontram protegidas pelo Código do Direito de Autor e Direitos Conexos e demais legislação aplicável, toda a cópia, parcial ou total, deste documento, nos casos em que é legalmente admitida, deverá conter ou fazer-se acompanhar por este aviso.

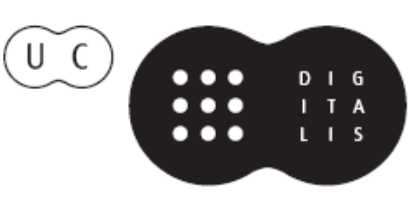



Tendo como pano de fundo as Comemorações dos seus 500 anos, a Biblioteca Geral da Universidade de Coimbra organizou um Congresso Internacional subordinado ao tema "A Biblioteca da Universidade: permanência e metamorfoses", que teve lugar nos dias 16, 17 e 18 de janeiro de 2014, no auditório da Reitoria da Universidade de Coimbra.

O objetivo maior desta reunião científica foi o de refletir sobre o presente e o futuro das bibliotecas que servem públicos universitários. Numa outra vertente, procurou chamar-se a atenção para a importância de que a Biblioteca se reveste, tendo em vista o progresso do conhecimento técnico e científico. Por último, o Congresso pretendeu instituir-se como oportunidade de reflexão prospetiva e como lugar de encontro entre as sensibilidades de todos os que trabalham profissionalmente com livros e com outros suportes de natureza bibliográfica.

Nesse sentido, foram apresentadas Conferências, Mesas Redondas e sessões de Testemunhos em torno de temas como o valor das bibliotecas universitárias, a biblioteca universitária em contexto; as mudanças e os desafios; a biblioteca universitária e a sociedade da informação e conhecimento; o impacto do acesso aberto na comunidade científica, e as bibliotecas digitais. 
ANTÓNIO TAVARES LOPES

Universidade de Coimbra

University of Coimbra

NOVAS ECOLOGIAS DO CONTROLO BIBLIOGRÁAICO:

UMA PERSPETIVA SOBRE OS FUTUROS DAS

\section{BIBLIOTECAS E DA INFORMAÇÃO BIBLIOGRÁFICA \\ NOS MEIOS D I G ITA I S}

NEW ECOLOGIES OF BIBLIOGRAPHIC CONTROL:

A PERSPECTICVE ON THE FUTURES OF BIBLIOGRAPHIC

INFORMATION AND DIGITAL LIBRARIES

RESUMO: Esta comunicação aborda os esforços de reconceptualização da informação bibliográfica nos atuais contextos de rede e de operacionalidade de sistemas de informação, e o seu contributo estratégico para o reposicionamento e as perceções de valor das bibliotecas, enquanto prestadoras de serviços informacionais. Recorrendo a uma observação de desenvolvimentos e iniciativas em curso na área do controlo bibliográfico, apresenta uma visão da biblioteca ou arquivo digital que não se esgota na transposição de suporte editorial, antes procura replicar e reconfigurar nos novos espaços de rede os princípios e o saber fazer da organização do conhecimento.

Palavras-chave: Controlo bibliográfico; FRBR; Web Semântica; Interoperabilidade; Bibliotecas digitais.

ABSTRACT: This paper addresses the efforts to reconceptualise bibliographic information within the current context of the network and the functionality of information systems, and their strategic contribution to the repositioning and perception of the value of libraries as providers of information services. Drawing on observations of ongoing developments and initiatives in bibliographic control, it presents a vision of the library or digital archive which is not restricted to transposing the editorial format, but instead seeks to replicate the principles and expertise involved in the organisation of knowledge within new networked spaces. 


\section{Introdução}

Creio ser meu dever começar por justificar o título desta comunicação e o evidente abuso dos plurais. Assumo que alarguei o alvo na esperança de fazer beneficiar a pontaria e o acerto. Olhando para a evolução recente das formas como produzimos e consumimos informação, no entanto, aceitável recorrer a esse cuidado, nesta exploração que o Congresso me dá a honra de poder partilhar, para tentar diminuir os desvios das aproximações por excesso ou por defeito que a singularidade mais facilmente origina. Os futuros, em vez do futuro, as bibliotecas, em vez da biblioteca, os meios digitais, em vez do meio digital.

Uma segunda justificação impõe-se, talvez até de forma mais urgente, pela introdução do termo "ecologia", também ele protegido pela maior indefinição do plural. Esta usurpação terminológica parecerá deslocada numa comunicação sobre controlo bibliográfico. No entanto, como espero poder saber salientar, o futuro, ou os futuros do controlo bibliográfico, serão marcados - estão a ser marcados - por uma profunda mudança no ambiente operacional da sua aplicação e na interação dos seus agentes com outros agentes, direi organismos, para sustentar a analogia ambiental, que não provêm da esfera biblioteconómica. Ecologia, portanto.

Apresentarei no final a minha perspetiva sobre o que considero ser razoável esperar, nos futuros percetíveis, da evolução da informação bibliográfica. Até lá chegar, farei o percurso que considero relevante para perceber as alterações ambientais mais significativas que sustentam aquelas previsões.

Recomeço, para isso, com um passo atrás: em 1998, ao mesmo tempo que o grupo de trabalho da IFLA que discutiu e preparou a revisão dos princípios do controlo bibliográfico publicava o documento de apresentação dos Functional Requirements for Bibliographic Records, o mundo da informação assistia a uma transformação sem precedentes impulsionada pela força emergente da World Wide Web.

A conceção do modelo dos FRBR resultou precisamente da tomada da consciência de que o ambiente de operação do controlo bibliográfico, o conjunto de procedimentos que descreve e identifica os materiais que 
são detidos ou referenciados pelas bibliotecas e agências de catalogação com vista ao acesso e à descoberta, se foi tornando cada vez mais rico e complexo ou, melhor dizendo, se foi tornando mais rica e complexa a compreensão das necessidades e solicitações dos utilizadores. E que seria necessário, em consequência, um modelo que soubesse representar melhor esse contexto de complexidade e responder aos seus desafios.

\section{Os serviços de informação bibliográfica e o impacto da Web}

A partilha, a troca e a comunicação de informação, que caracterizam de forma basilar a própria ideia da World Wide Web, nunca foram estranhas às bibliotecas. Bem pelo contrário, fazem vincadamente parte da sua matriz fundadora.

Com a inscrição na gestão das bibliotecas de sistemas de informação de base tecnológica, a ideia de partilha deixa de se restringir aos materiais que são objeto do controlo bibliográfico e passa a estender-se à partilha da própria informação sobre esses materiais, àquilo que hoje em dia genericamente designamos, no espaço da rede, por metadados. Se os destinatários da partilha e comunicação dos materiais são as comunidades que servem, os da partilha da informação de controlo começam por ser as próprias bibliotecas constituídas mais ou menos informalmente em redes de âmbito geográfico e tipológico diversificado. Catalogar apenas uma vez e replicar o registo bibliográfico por todas as agências interessadas foi um objetivo generosamente abraçado pela comunidade de bibliotecas e que, devido ao seu investimento, se tornou uma operação de processos formalizáveis e de elevada razoabilidade económica.

Os primeiros instrumentos utilizados para este efeito, e que ainda hoje respondem por grande parte dos procedimentos de troca e comunicação de informação bibliográfica entre bibliotecas e agências catalogadoras, foram concebidos para operarem num ambiente tecnológico específico. A norma de transferência de dados bibliográficos de maior sucesso e capacidade de sobrevivência, que se viria a fixar numa norma ISO - a 2709, foi desenhada para a comunicação em fita magnética e para um ambiente de operação desconectado em que a intervenção humana direta 
é fundamental para garantir o sucesso das transações, começando pelo próprio transporte e manipulação dos materiais de suporte. No que a este aspeto interessa, pode dizer-se que a principal e significativa diferença entre esse ambiente tecnológico e o que atualmente predomina é precisamente a introdução hegemónica das aplicações da Internet, em particular a World Wide Web. Esta hegemonia é sustentada pela integração de redes supralocais e supraorganizacionais e pelo desenvolvimento e maturação de várias camadas de protocolos de comunicação entre sistemas que se formalizam na proporção inversa da necessidade de supervisão humana direta. Para o controlo bibliográfico, como para muitos outros processos e domínios técnicos e do conhecimento, esta alteração do ambiente tecnológico significa a necessidade de se ajustar à presença de forças de sentido distintos mas concorrentes: por um lado, a proliferação de fontes e polos de informação material e imaterial reunidos num meio informacional mais ou menos integrado e, por outro, a facilitação e aumento dos meios e fluxos de troca de informação que abrem novos caminhos e oportunidades à exploração da interoperabilidade.

Estas novas condições ambientais em que os sistemas programados assumem predominância, implicam novos padrões de comportamento dos diversos agentes envolvidos. O mais radical no que às bibliotecas importa - e em grande parte preocupa - é que elas viram diminuir dramaticamente os níveis de fidelidade e de exclusividade dos seus utilizadores. Expostos agora a um ambiente desenhado para combinar agilmente a referência, o acesso e o consumo de informação, os tradicionais e potenciais utilizadores das bibliotecas têm razões objetivas para privilegiar e recompensar um comportamento a que atribuem elevadas taxas de sucesso. Este comportamento tende a marginalizar as soluções mediadoras que obrigam a procedimentos adicionais e exteriores aos instrumentos de referência para consumo da informação. Ou seja, os procedimentos que são tradicionalmente os das bibliotecas ${ }^{1}$.

1 OCLC - Perceptions of libraries and information resources. Dublin (Ohio): OCLC, 2005. ISBN 1-55653-364-0. OCLC - Perceptions of libraries: context and community. Dublin (Ohio): OCLC, 2010. ISBN 1-55653-395-0. 
Deixarei de lado a caracterização de algumas respostas das bibliotecas a este problema, que têm sido e continuarão a ser objeto de comunicações neste Congresso, e concentrar-me-ei no que mais toca à informação bibliográfica.

\section{Os FRBR e a reconceptualização do controlo bibliográfico}

Num primeiro passo, é a definição dos requisitos funcionais dos registos bibliográficos, identificada pela sigla FRBR, que representa o esforço mais sistemático e estruturante de revisão da conceptualização da informação bibliográfica, incidindo sobre a sua formação e função. Ou seja, sobre o controlo bibliográfico e a aplicação do seu produto na identificação e satisfação das necessidades de pesquisa e exploração de informação dos utilizadores. Conceptualmente, o desenho dos FRBR serve o propósito de redefinir a abordagem e perspetiva das tarefas e funções do controlo bibliográfico e, por isso, situa- se a um nível de abstração não necessariamente próximo da implementação que os sistemas de informação bibliográfica deverão concretizar² .

Os FRBR manifestam-se, em primeiro lugar, como um modelo conceptual da informação bibliográfica baseado no modelo relacional Entidade-Relação, em que as diversas entidades se distribuem por três grupos: no primeiro, reúnem-se as entidades que representam os produtos do esforço intelectual e artístico; no segundo, acrescentam-se as que representam as responsabilidades na criação, produção e custódia das entidades do primeiro grupo; no terceiro, a representação como assunto que descreve ou contextualiza as entidades do segundo.

O modelo relacional que orienta a criação dos FRBR não está de todo ausente na formalização dos modelos de dados definidos anteriormente para a expressão das normas bibliográficas patrocinadas pela IFLA, com

2 IFLA - Functional requirements for bibliographic records: final report. 1997 (revisão até 2009). [Em linha]. [Consult. 3 nov. 2013]. Disponível na WWW em: <URL:http://www. ifla.org/files/assets/cataloguing/frbr/frbr_2008.pdf>. Versão portuguesa: Registos funcionais dos registos bibliográficos. Lisboa: Biblioteca Nacional de Portugal, 2008. 
o UNIMARC, e pela Library of Congress, com o MARC21, e que se tornaram dominantes a nível internacional. Em ambos os casos encontram-se reguladas normas de registo específicas para o controlo bibliográfico, para o controlo de autoridade, para a classificação e para o controlo de existências, estabelecendo-se entre estas diversas entidades (para usar uma terminologia do modelo relacional) associações explícitas determinísticas, ainda que pouco robustas tendo em conta o seu caráter opcional e a predisposição para a redundância não controlada.

Os FRBR vêm introduzir duas revisões fundamentais a esta modelação, operando diretamente sobre o nível do registo do controlo bibliográfico (antes ainda de considerar, portanto, a dimensionalidade que o uso articulado das restantes normas autorizava e que desenvolvimentos do novo modelo mantêm mas que não serão aqui referidas). Em primeiro lugar, os FRBR formalizam conceptualmente um conjunto muito diferenciado e aprofundado de entidades, libertando-as dos limites horizontais do registo bibliográfico tradicional ao mesmo tempo que clarificam e definem as relações que estabelecem entre si; em segundo lugar, e como corolário da primeira revisão, alteram o foco de atenção do controlo bibliográfico do item para a obra, isto é, do exemplar que está a ser descrito para a criação intelectual ou artística de que é uma representação ou, precisando, para a cadeia da criação intelectual e artística que começa na obra e se pode materializar, finalmente, no item.

Segundo os FRBR, estas necessidades são expressas num conjunto de quatro operações genéricas que incidem sobre as entidades definidas pelo modelo, considerando os atributos específicos de cada uma e as relações que estabelecem com as outras entidades. O utilizador pode encontrar entidades em resultado da formalização de uma pesquisa; identificar uma entidade em relação a outras; selecionar uma entidade adequada às suas condições de consumo de informação; e obter a informação e o conteúdo que lhe interessa na forma que lhe interessa.

É para responder a estas operações genéricas que o novo modelo conceptual torna discretas as diferentes dimensões do material controlado bibliograficamente: ao modelar a informação bibliográfica de maneira a sugerir formalmente a construção de um conjunto de declarações que 
exprimem relações entre entidades conceito e a sua recuperação por um mesmo processo formal, os FRBR procuram que os sistemas de informação bibliográfica ascendam da estrita função de catálogo ou repositório de materiais controlados para uma base de conhecimento sobre estes materiais e sobre o seu contexto de criação e produção.

\section{Implementação dos FRBR: avanços e dificuldades}

A mudança de paradigma que recoloca o utilizador como sujeito da atividade do controlo bibliográfico e a criação intelectual ou artística como o seu objeto, e que visa reforçar o valor intrínseco e percebido da biblioteca, ainda que alicerçada numa tradição de conceitos e procedimentos, está, no entanto, longe de encontrar eco real significativo nas nossas práticas de catalogação. Aquilo que se designa por "frbrização" do catálogo - o processo que migra a informação bibliográfica do modelo de dados tradicional para um novo modelo, derivado do modelo conceptual dos FRBR - não ganhou ainda o impulso necessário para se tornar prática generalizada e alterar as condições técnicas de execução do controlo bibliográfico, apesar de algumas iniciativas salientes.

Uma destas iniciativas relaciona-se com a redefinição das regras anglo-americanas de catalogação, as AACR2, pensada de forma a poder suportar os princípios modelares dos FRBR. Este trabalho resultou na produção das regras RDA (Resource Description and Access) cujos testes de aplicabilidade decorreram recentemente num conjunto de instituições selecionadas. Uma das conclusões destes testes é a dificuldade em recorrer às normas tradicionais para exprimir a informação bibliográfica segundo a RDA, o que levou ao lançamento da Bibliographic Framework Transition Initiative, um projeto que visa a substituição do MARC21 como transportador da informação bibliográfica ${ }^{3}$.

3 Sítio Web desta iniciativa em: LIBRARY OF CONGRESS - BIBFRAME: Bibliographic Framework Initiative. [Em linha]. Washington, DC: LOC, [s.d.]. [Consult. 3 nov. 2013]. Disponível na WWW em: <URL:http://www.loc.gov/bibframe/>. 
Os mesmos sentimentos e preocupações obrigaram a IFLA a olhar para a sua norma UNIMARC e propor uma revisão que responda a algumas das necessidades de codificação dos FRBR e modelos associados. Essa revisão será motivo para um nova versão da norma, em substituição da corrente de 2008, pontualmente revista com aquele propósito em mente, mas não se estranhará se uma remodelação mais radical e orientada para os fundamentos dos FRBR venha a acontecer, tal como se prevê para o MARC214.

A perspetiva e desenvolvimento destas redefinições têm sido acompanhados por iniciativas de carácter mais ou menos experimental mas de aplicação imediata, como as da OCLC5, destacando-se o FictionFinder, uma ferramenta de exploração do seu WorldCat segundo os princípios dos FRBR.

As razões para as dificuldades da "frbrização" decorrem, em primeiro lugar, e de um ponto de vista puramente técnico, da inadequação dos atuais modelos de dados bibliográficos para funcionarem como suporte aos novos modelos conceptuais. Nada impediria, no entanto, que este processo se baseasse em soluções proprietárias ou autónomas, como, aliás, decidiu a OCLC para o desenvolvimento das suas iniciativas. Mas existe igualmente um outro tipo de barreira, decorrente da ausência de uma recompensa percetível que justifique o esforço, numa altura em que os recursos técnicos à disposição das bibliotecas têm a vindo a diminuir e a contração do acesso a novos meios e a mais apoio é generalizada6 .

Em alternativa às respostas top-down, cuja eficácia, ainda que se possa vir a verificar, não será facilmente transportável para todos os contextos

4 LE PAPE, Philippe - Expressing FRBR in UNIMARC: Yes we can! In World Library and Information Congress: 77th IFLA General Conference and Council. San Juan (Porto Rico), 2011. [Em linha]. [Consult. 3 nov. 2013]. Disponível na WWW em: <URL:http://conference. ifla.org/past/ifla77/187-pape-en.pdf>.

5 Informação sobre as iniciativas da OCLC em OCLC RESEARCH - OCLC research activities and IFLA's Functional Requirements for Bibliographic Records [Em linha]. [Consult. 3 nov. 2013]. Disponível na WWW em: <URL:http://www.oclc.org/research/activities/frbr.html>.

6 GRADMANN, Stefan - rdfs:frbr-Towards an implementation model for library catalogs using semantic Web technology. Cataloging \& Classification Quarterly. 39:3-4 (2005) 63-75. ŽUMER, Maja - FRBR: the end of the road or a new beginning? Bulletin of the American Society for Information Science and Technology. 33-6 (2007) 27-29. 
nacionais ou organizacionais, cresceu e solidificou-se entre os promotores dos FRBR a ideia de que existe uma oportunidade de valorização e recompensa para esta reconceptualização da informação bibliográfica no espaço da rede mundial de informação digital. Esta oportunidade, cujo programa se pode sintetizar na expressão feliz de Shawne Miksa, "we need to "connect", and not just "represent"»", exige na sua base que se dê um novo passo nas atitudes de partilha, não já com os seus utilizadores, como esteve na fundação das bibliotecas, não já com as seus congéneres, como acompanhou o seu processo de informatização, mas agora indiferenciadamente com os diversos sistemas e serviços de informação no espaço da Web $^{8}$.

\section{A Web Semântica e o novo espaço da troca de informação}

O campo de concretização desta oportunidade que se encontra atualmente mais sistematizado é definido pelo movimento que, do lado da Web e sob a bandeira do consórcio W3C, procura criar as condições para a formalização da troca de informação entre sistemas. A Web Semântica é, em primeiro lugar, uma "web of data", ou mais precisamente, uma "web of linked data", um espaço onde dados e o seu contexto semântico são identificados segundo um conjunto de regras estruturais comuns, de forma a poderem ser processáveis por sistemas automáticos.

Peço que entendam esta pequena introdução à Web Semântica com interesse mais declarativo do que explicativo, e apenas no que espero

7 MIKSA, Shawne D. - Introduction. Journal of Library Metadata. 12:2-3 (2012) 53-60.

8 GREENBERG, Jane - Advancing the Semantic Web via library functions. Cataloging $\mathcal{E}$ Classification Quarterly. 43:3-4 (2007) 203-225. DUNSIRE, Gordon - UNIMARC, RDA and the Semantic Web. In World Library and Information Congress: 75th IFLA General Conference and Council. Milão, 2009. [Consult. 3 nov. 2013]. Disponível na WWW em: <URL:http:// conference.ifla.org/past/ifla75/135-dunsire-en.pdf>. DUNSIRE, Gordon; HILLMANN, Diane; PHIPPS, Jon - Reconsidering universal bibliographic control in light of the semantic Web. Journal of Library Metadata. 12:2-3 (2012) 164-176. HOWARTH, Lynne C. - FRBR and linked data: connecting FRBR and linked data. Cataloging E Classification Quarterly. 50:5-7 (2012) 763-776. COYLE, Karen - Linked data tools: connecting on the Web. Chicago: American Library Association, 2012. (Library Technology Reports). 
ser suficiente para me assistir na clarificação do reconhecimento de oportunidade que mencionei acima.

O principal instrumento para a construção da Web Semântica é o RDF (de Resource Description Framework), um modelo gramatical que define expressões na forma sujeito-predicado-objeto para descrever os vários elementos de informação que pertencem a uma qualquer base de conhecimento, independentemente da sua dimensão, âmbito, nível de agregação ou outras características que os distingam. Estas expressões têm o nome de triple e associam a um recurso (a coisa sobre a qual sabemos algo) uma propriedade com determinado valor observado, e que é declarado9. Por exemplo: "este livro" (sujeito) "tem autor" (predicado) "Fernando Pessoa" (objeto).

A pretendida processabilidade dos dados deve entender-se no quadro operacional da comunicação entre sistemas, e a um nível superior ao da simples compreensão formal da mensagem ou do repositório: considerando dois programas como os pontos - originador e recetor - na comunicação síncrona ou assíncrona de informação na Web Semântica, o modelo RDF serve o propósito de garantir que o recetor, percebendo a estrutura da mensagem ou dos dados, perceba também a informação que estes carregam: a informação é, assim, legível e processável.

A capacidade de inteligibilidade dos agentes depende do acesso a uma rede de conhecimentos em domínios relevantes e à competência no uso das ferramentas formais de processamento e comunicação. A acompanhar a regulação dos instrumentos formais, de que o RDF é apenas o ponto de partida, tem sido levado a cabo um grande esforço no sentido de formalizar domínios do conhecimento de maneira que possam ser usados por sistemas computacionais, em particular no quadro da Web Semântica (mas não se limitando aí). Esquemas de metadados, linguagens e vocabulários que exprimem as diversas propriedades que, na perspetiva de um determinado domínio ou de um modo geral de representação, servem

9 KLYNE, Graham; CARROLL, Jeremy, eds. - Resource Description Framework (RDF): concepts and abstract syntax. Versão: fevereiro de 2004. [Consult. 3 nov. 2013]. Disponível na WWW em: <URL: http://www.w3.org/TR/2004/REC-rdf-concepts-20040210/>. 
para descrever os recursos - as coisas - que esse domínio consegue compreender, estão a ser criados, desenvolvidos, sistematizados e publicados.

A identificação por Uniform Resource Identifiers (URI) de nomes, objetos, factos, conceitos, propriedades, regras, classes, tipos, todo o conjunto de elementos sobre os quais se formam estes instrumentos, ajuda a manter organizadas as correntes de informação e a garantir entre os sistemas agentes da Web Semântica a transportabilidade e comunicabilidade do conhecimento que temos sobre o mundo, segundo o foco que cada domínio representa. Idealmente, estes identificadores, estes URI, devem ser resolvidos de forma a serem distintamente utilizados por agentes informáticos e humanos.

Sobre estes três instrumentos da Web Semântica - descrição, domínios semânticos, identidade - começa a ser possível fazer aumentar a capacidade para interligar dados e gerar novo conhecimento, aqui entendido como a determinação de informação que não se encontra explícita em qualquer banco de dados, mas que pode ser inferida a partir do cruzamento de vários.

\section{A Web Semântica como oportunidade de afirmação da reconcep- tualização do controlo bibliográfico}

A "rdfização" do catálogo bibliográfico - para utilizar um outro jargão recente que identifica o processo pelo qual determinada informação passa a ser expressa segundo declarações RDF - poderá vir a recorrer a esquemas de metadados já estabelecidos para incrementar a sua inteligibilidade potencial, quer pela reutilização de classes e tipos que se encontram já definidos em outros namespaces (isto é, um contexto de identificadores únicos) quer pela aplicação de redundância na expressão dos dados: a utilização da classe Person e das suas propriedades do vocabulário FOAF para tipificar a classe Person num catálogo FRBR seria um possível exemplo do primeiro; o acompanhamento do atributo title da instância de um Work num catálogo FRBR por uma instância da propriedade title do Dublin Core um exemplo do segundo. 
Deixando de lado algumas propostas de carácter mais experimental, quero registar duas intervenções na dinâmica da Web Semântica que emanam da área do controlo bibliográfico e que, podendo igualmente ser entendidas como de natureza propedêutica, pelo seu carácter fundacional terão certamente impacto significativo no sequente desenvolvimento de esquemas e sistemas para operar nesse espaço.

Por um lado, tem-se procurado registar formalmente os identificadores de classes, propriedades, tipos e enumerações que integram os diversos modelos de organização e representação da informação bibliográfica. No Open Metadata Registry ${ }^{10}$, e no momento em que apresento esta comunicação, encontram-se registados vocabulários relativos ao modelo conceptual dos FRBR, ao modelo de representação da ISBD, aos elementos do sistema de descrição da RDA e mesmo a alguns componentes do MARC21. Mesmo que este esforço venha a necessitar de revisão, ilustra claramente um dos caminhos que terá de ser trilhado para a inscrição do controlo bibliográfico no espaço da Web Semântica.

Por outro lado, sob os auspícios da OCLC criou-se um sistema de autoridades a nível global, o Virtual International Authority File (VIAF) ${ }^{11}$, que reúne as contribuições de um conjunto de organizações de referência e autoridade bibliográfica, entre as quais várias bibliotecas nacionais. Este sistema, que cobre a diversidade de representações de títulos e nomes referenciados em sistemas de informação bibliográfica, proporciona a identificação única desses diversos títulos e nomes no espaço da Web, e associa-os às formas preferenciais de representação tal como os diferentes agentes contribuintes as manifestaram.

O desenvolvimento destas iniciativas tornará possível fazer sair os processos e os produtos do controlo bibliográfico do restrito âmbito biblioteconómico. Neste caminho, e concluindo as diversas direções que nesta comunicação pretendi reunir, tentarei antecipar alterações significa-

10 Serviço de referência disponível em METADATA MANAGEMENT Associates - Open metadata registry. [consult. 3 nov. 2013]. Disponível na WWW em: <URL:http://metadataregistry. $\operatorname{org} />$.

11 Serviço de referência disponível em OCLC - VIAF: virtual international authority file. 2010-2014. [consult. 3 nov. 2013]. Disponível na WWW em: <URL:http://viaf.org/>. 
tivas no futuro da informação bibliográfica. Não se trata de um programa de ação, muito menos de um conjunto de conselhos, que viria sempre a despropósito apresentar a uma Casa que celebra os seus 500 anos de vida.

O que podemos esperar, então, do futuro do controlo bibliográfico?

- Assistiremos a uma desagregação do registo bibliográfico, transformado numa rede ou grafo de declarações informacionais elementares que relacionam objetos e conceitos tanto quanto os descrevem - esta desagregação ocorrerá no nível da organização da informação por parte dos sistemas, atualmente mapeados para as diversas normas MARC, mas será igualmente operacional nos processos de comunicação, para agentes humanos e computacionais. Em conformidade, as normas MARC serão redesenhadas - elas próprias desagregadas num processo que corresponderá efetivamente a uma substituição - de forma a representar mais facilmente o carácter compósito da informação bibliográfica, segundo os princípios dos FRBR e dos seus desenvolvimentos. - Corolário do ponto anterior, a formatação da informação bibliográfica será muito mais neutra relativamente a aplicações específicas. Por exemplo, veremos desaparecer a contaminação pela pontuação. A produção da descrição bibliográfica passará a ser resultado de um processamento particular dos diversos elementos relacionados com determinada expressão de uma obra.

- A informação bibliográfica extravasará do catálogo e será reutilizada em sistemas de informação e conhecimento, incluindo a biblioteca digital. Contribuirá significativamente para o enriquecimento semântico das bibliotecas digitais, ultrapassando os limites a que hoje assistimos da simples transposição editorial do impresso para o digital, ou de outros suportes analógicos para o digital. Aliás, a lógica do objeto impresso, na sua simulação do físico e na sua unidirecionalidade, impera em muitas das atuais bibliotecas digitais, mesmo quando os objetos são nado digitais. O enriquecimento por via do controlo bibliográfico, de descrição e de análise de conteúdo, será sobretudo relevante no texto científico e permitirá introduzir valor diferenciado nas bibliotecas digitais. 
- Em consequência, onde possível, catálogo bibliográfico e biblioteca digital convergirão a ponto de se confundirem: a informação bibliográfica integrará ou estará muito próxima dos objetos digitais e os sistemas de exploração e consumo reconhecerão nessa convergência o potencial para conduzir os agentes consumidores para informação adicional relevante.

- O controlo de autoridades será generalizado e assumido como um procedimento prioritário, ou melhor, fundamental, decorrendo da remodelação da informação bibliográfica inspirada pelos FRBR, e inscrito com particular importância na relação das bibliotecas com as suas comunidades (em particular, a académica). Ainda que local, este controlo tenderá a ser mapeável para estruturas globais de informação e conhecimento.

- Aprofundando: no catálogo bibliográfico tudo será controlo de autoridade. Ou seja, os processos de catalogação, indexação, classificação, serão traduzidos na identificação das propriedades de um determinado objeto nos diversos domínios, nos diversos namespaces, para utilizar o conceito da Web. Usando uma expressão feliz de Richard Wallis, deixaremos de catalogar, passaremos a cataligar. Catalinking, instead of cataloging.

- A informação bibliográfica será abundante, aberta e livre, normalmente disponibilizada segundo uma licença Creative Commons CC0, e cada vez mais presente, por via direta ou indireta, nos processos de consumo e organização de informação dos diversos públicos.

\section{Conclusão}

A associação da informação bibliográfica à construção da Web Semântica representa uma oportunidade que encontra as suas razões não apenas na expectativa de uma valorização, mas sobretudo na inscrição formal do controlo bibliográfico como simultaneamente contribuinte e beneficiário dos sistemas de conhecimento em rede digitais. 
Ao inserir-se neste movimento coletivo, ao disponibilizar um produto do seu conhecimento técnico em condições de ser reutilizado, as bibliotecas, em primeiro lugar, inscrevem-se em diversas cadeias de valor do negócio da informação, aumentando a sua visibilidade e relevância; depois, beneficiam do sentido inverso do movimento, ou seja, instalam-se num ambiente muito rico do ponto de vista informacional e do ponto de vista da qualidade dos processos; finalmente, permite que estabeleçam e defendam um padrão de procedimentos cuja qualidade se torna reconhecível fora da sua área específica de negócio.

Ao valorizar a representação da criação intelectual e artística e da cadeia de produção que, finalmente, ganha corpo num item concreto, em detrimento da conceção tradicional de relevar essa materialização, a reconceptualização na formação e aplicação do controlo bibliográfico corresponde muito melhor à nossa perceção da complexidade e da cadeia de conexões que estão na origem dos processos de conhecimento e cultura da humanidade e que hoje encontram, na Web, novo campo de expressão.

\section{Referências bibliográficas}

CAlHoun, Karen; REgister, Renee - Next generation cataloging. Journal of Library Administration, 49:6 (2009) 651-656.

COYLE, Karen - Linked data tools: connecting on the Web. Chicago: American Library Association, 2012. (Library Technology Reports).

DANSKIN, Alan - "Tomorrow never knows": the end of cataloguing? IFLA Journal. $33: 3$ (2007) 205-208.

DUNSIRE, Gordon - UNIMARC, RDA and the Semantic Web. [Em linha]. In World Library and Information Congress: 75th IFLA General Conference and Council. Milão: IFLA, 2009. [Consult. 3 nov. 2013]. Disponível na WWW em: <URL:http:// conference.ifla.org/past/ifla75/135-dunsire-en.pdf.>.

DunsiRe, Gordon; HillmanN, Diane; Phipps, Jon - Reconsidering Universal Bibliographic Control in light of the Semantic Web. Journal of Library Metadata. 12:2-3 (2012) 164-176. 
GARDNER, Sue Ann - The changing landscape of contemporary cataloging. Cataloging E Classification Quarterly. 45:4 (2008) 81-99.

GRADMANN, Stefan - rdfs:frbr-towards an implementation model for library catalogs using semantic Web technology. Cataloging \& Classification Quarterly. 39:3-4 (2005) 63-75.

GREENBERG, Jane - Advancing the semantic Web via library functions. Cataloging E Classification Quarterly. 43:3-4 (2007) 203-225.

HICKEY, Thomas B.; O'NEILL, Edward T. - FRBRizing OCLC's WorldCat. Cataloging E Classification Quarterly. 39:3-4 (2005) 239-251.

HOWARTH, Lynne C. - FRBR and linked data: connecting FRBR and linked data. Cataloging \& Classification Quarterly. 50:5-7 (2012) 763-776.

KLYNE, Graham; CARROLL, Jeremy (eds.) - Resource description framework (RDF): concepts and abstract syntax. Versão: fevereiro de 2004. [Em linha]. [Consult. 3 nov. 2013]. Disponível na WWW em: <URL:http://www.w3.org/TR/2004/ REC-rdf-concepts-20040210/>.

IFLA - Functional requirements for bibliographic records: final report. 1997 (revisão até 2009). [Em linha]. [Consult. 3 nov. 2013]. Disponível na WWW em: <URL:http://www.ifla.org/files/assets/cataloguing/frbr/frbr_2008.pdf>.

IFLA - Versão portuguesa: Registos funcionais dos registos bibliográficos. Lisboa: Biblioteca Nacional de Portugal, 2008.

LE PAPE, Philippe - Expressing FRBR in UNIMARC: Yes we can! In World Library and Information Congress: 77th IFLA General Conference and Council. San Juan (Porto Rico), 2011. [Em linha]. [Consult. 3 nov. 2013]. Disponível na WWW em: <URL:http://conference.ifla.org/past/ifla77/187-pape-en.pdf>.

LIBRARY OF CONGRESS - BIBFRAME: Bibliographic Framework Initiative. [Em linha]. Washington, DC: LOC, [s.d.]. [Consult. 3 nov.]. Disponível na WWW em: <URL:http://www.loc.gov/bibframe/>.

METADATA MANAGEMENT ASSOCIATES - Open metadata registry. [consult. 3 nov. 2013]. Disponível na WWW em: <URL:http://metadataregistry.org/>.

MIKSA, Shawne D. - Introduction. Journal of Library Metadata. 12:2-3 (2012) 53-60.

NAUN, Chew Chiat - Next generation OPACs: a cataloging viewpoint. Cataloging E Classification Quarterly. 48:4 (2010) 330-342.

OCLC - Perceptions of libraries and information resources. Dublin (Ohio): OCLC, 2005. ISBN 1-55653-364-0. 
OCLC - Perceptions of libraries, 2010: context and community. Dublin (Ohio): OCLC, 2010. ISBN 1-55653-395-0.

OCLC - VIAF: virtual international authority file. 2010-2014. [consult. 3 nov. 2013]. Disponível na WWW em: <URL:http://viaf.org/>.

OCLC RESEARCH - OCLC research activities and IFLA's Functional Requirements for Bibliographic Records [Em linha]. [Consult. 3 nov.]. Disponível na WWW em: <URL:http://www.oclc.org/research/activities/frbr.html>.

ROSE, Mary Z. - The ship has sailed and we aren't on it: how catalogers could support user tasks and why we won't. Journal of Library Metadata. 12:2-3 (2012) 127-139.

WORLD WIDE WEB CONSORTIUM (W3C) - W3C Semantic Web activity. [Em linha]. [Consult. 3 nov. 2013]. Disponível na WWW em: <URL:http://www.w3.org/2001/ $\mathrm{sw} />$.

ŽUMER, Maja - FRBR: the end of the road or a new beginning? Bulletin of the American Society for Information Science and Technology. 33-6 (2007) 27-29. 
José Augusto Cardoso Bernardes é Professor da Faculdade de Letras da Universidade de Coimbra e Diretor da Biblioteca Geral da Universidade

Ana Maria Eva Miguéis é coordenadora do Serviço Integrado das Bibliotecas da Universidade de Coimbra

Carla Ferreira é bibliotecária nos Serviços de Biblioteca e Documentação da Faculdade de Letras da Universidade de Coimbra. 


\section{Série Documentos}

Imprensa da Universidade de Coimbra

Coimbra University Press

2015

C •

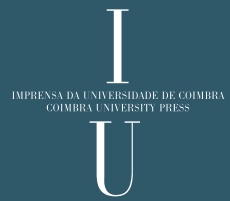

